\title{
Largest Problem: Getting a crystal and what is it worth to the researcher to have the 3D crystalline structure? Academic and Industrial Aspects. \\ R Staples ${ }^{1}$ \\ ${ }^{1}$ Dept of Chemistry, Michigan State University staples@chemistry.msu.edu
}

This talk will focus on some of the non-traditional aspects that come up when running a crystallographic facility for both academic research and industrial settings. What are the anticipated costs and what are the hidden pit falls that can derail your budget? It is hoped that it will highlight some of the philosophic differences in aspects of running a crystallographic laboratory in the different settings. 